\title{
Fatores de Risco de Aterosclerose na Infância. Um Estudo Epidemiológico
}

\author{
Zoffi Roberto S. Gerber, Paulo Zielinsky \\ Porto Alegre, RS
}

Objetivo - Obter um perfil dos fatores de risco coronário em uma amostra populacional pediátrica da cidade de Bento Gonçalves, $R S$, no período de maio/90 a junho/91.

Métodos - Foram estudados 1501 escolares de 6 a 16 anos incompletos, visando a detecção dos níveis séricos de colesterol total, lipoproteínas, triglicerídeos, bem como a avaliação da pressão arterial e da história familiar de doença cardiovascular isquêmica e obesidade.

Resultados - Foram detectadas $420(27,98 \%)$ crianças com hipercolesterolemia, sendo que $75(5 \%)$ apresentavam hipertensão arterial sistólica e 48 (3,20\%) hipertensão arterial diastólica. A história familiar foi importante quando positiva, porém, sua ausência não excluia a presença de fatores de risco para a aterosclerose. A hipertrigliceridemia foi encontrada em $136(9,06 \%)$ escolares e a LDL-colesterol elevada em 155 (10,33\%), mostrando forte associação com hipercolesterolemia. Apresentaram indice de massa corporal acima de percentil 95, não mostrando uma maior prevalência de hipercolesterolemia, 111 crianças.

Conclusão - Os fatores de risco para a aterosclerose estão presentes na infância e deveriam ser pesquisados independentemente do nível socioeconômico, da história familiar, da idade e do sexo, devendo o pediatra ser um dos responsáveis por esta investigação.

Palavras-chaves: doenças cardiovasculares, arteriosclerose coronária, fatores de risco, criança

\section{Risk Factors of Atherosclerosis in Children. An Epidemiologic Study}

Purpose - To obtain a profile of risk factors for coronary atherosclerosis in a pediatric population from Bento Gonçalves, Rio Grande do Sul, from May 1990 to June 1991.

Methods - One thousand five hundred and one children with ages from 6 to 15 years were studied to assess serum cholesterol levels and their association with other risk factors, such as arterial hypertension, obesity and family history of cardiovascular disease.

Results - Four hundred and twenty (27.98\%) children showed cholesterol levels over 180mg\%; 75 (5\%) had systolic hypertension and $48(3.20 \%)$ diastolic hypertension. The family history was important to search for riskfactors such as hypercholesterolemia, but did not exclude risk factors when negative. One hundred and thirty six $(9.06 \%)$ children showed hipetriglyceridemia; 155 (10.33\%) presented LDL-cholesterol above $130 \mathrm{mg} \%$ and showed a strong association with hypercholesterolemia. Obesity was present in 111 children, but none showed a major prevalence of hypercholesterolemia.

Conclusion - Risk factors for atherosclerosis are present in children and their search should be performed, independent of family income, family history, age and sex. The pediatrician must have a significant role in this setting.

Key-words: cardiovascular disease, atherosclerosis, risk factors, child

Arq Bras Cardiol, volume 69 (n 4), 231-236, 1997

As doenças cardiovasculares destacam-se, nos dias atuais, como a mais freqüente causa de óbito ${ }^{1}$. A patogenia mais encontrada das doenças cardiovasculares é, indiscuti-

Instituto de Cardiologia do Rio Grande do Sul/Fundação Universitária de Cardiologia - Porto Alegre

Correspondência: Zoffi Gerber - Unidade de Pesquisa - Instituto de Cardiologia do RS - Av. Princesa Isabel, 395 - 90620-001 - Porto Alegre, RS

Recebido para publicação em 23/5/97

Aceito em 29/7/97 velmente, a aterosclerose coronária, que pode acometer, inclusive, pacientes jovens ${ }^{2}$. Monckberg, citado por Cresanta e $\mathrm{col}^{3}$, examinou aortas de crianças que faleceram durante a $1^{a}$ guerra mundial e, em 1921, relatou que $36 \%$ de jovens com menos de 20 anos e $76 \%$ dos que tinham entre 20 e 25 anos, por ocasião do óbito, apresentavam ateromatose da íntima da aorta. Anos após, Zeek, em 1930, citou que a aterosclerose ocorre em qualquer idade ${ }^{4}$.

Holman e $\mathrm{col}^{5}$ relataram, em 1958, que todas as crian- 
ças com três anos de idade ou mais já eram portadoras de estrias de gordura em aorta e que todas as pessoas com 20 ou mais anos de idade tinham estrias gordurosas nas artérias coronárias. Estas lesões, vistas em crianças, estão diretamente relacionadas com as lesões em relevo observadas mais tarde ${ }^{6}$.

A incidência de coronariopatia é, em geral, dependente da prevalência de seus fatores de risco. Quanto maior a presença de fatores de risco para a aterosclerose, maior a probabilidade de incidir uma coronariopatia.

Dentre os fatores de risco mais freqüentes estão a hipercolesterolemia, o hábito de fumar, a hipertensão arterial (HA), a hipertrigliceridemia, o excesso de peso e a história familiar de cardiopatia isquêmica.

Embora os fatores de risco para aterosclerose estejam presentes desde os primórdios da vida, são poucos os estudos destinados a avaliá-los na idade pediátrica.

Este trabalho teve como objetivo a obtenção do perfil do colesterol sérico em uma amostra pediátrica, para a identificação de grupos com risco potencial aumentado para doença cardíaca coronária (DAC), verificando a associação do nível sérico do colesterol com a presença de outros fatores de risco coronário, analisando a relação do valor sérico do colesterol de acordo com a faixa etária, o sexo, a raça e o nível socioeconômico e estudando as relações entre os valores elevados de colesterol na amostra e níveis séricos de triglicerídios e lipoproteínas.

\section{Métodos}

Nosso estudo, segundo os eixos de delineamento de pesquisa, constou de modo comparado simultâneo, individual e contemporâneo, caracterizando pesquisa do tipo transversal ou de prevalência.

Foram estudados 1501 indivíduos, com idades variando de 6 a 16 anos incompletos, todos escolares e residentes em Bento Gonçalves (RS), de maio/90 a junho/91.

De acordo com dados oficiais da Secretaria de Educação do município, Bento Gonçalves possui 11.793 estudantes ${ }^{7}$ e participaram do estudo 1501 , correspondendo a $12,73 \%$ da população estudantil.

A coleta dos dados, primeiramente, constou de um contato com cada uma das direções das escolas particulares e da rede pública, visando sensibilizá-las sobre a frequiência dos fatores de risco para a aterosclerose. Obtida a concordância, era enviado um convite aos pais para que participassem, nos respectivos colégios, de uma palestra, onde era mostrada a prevalência dos fatores de risco coronário em jovens, através de estudos internacionais. Os pais ou responsáveis recebiam um formulário com o qual poderiam ou não concordar que seus filhos fossem investigados. Obtida a autorização, entregue por escrito, os escolares eram convidados a comparecer na escola, na parte da manhã, após um jejum prévio de no mínimo $12 \mathrm{~h}$, para a coleta dos exames laboratoriais (colesterol, HDL, LDL, triglicerídios) e só depois, era feito o exame médico.

$\mathrm{O}$ exame médico constava de uma anamnese dirigida através do preenchimento de um questionário individual e também do exame físico, no qual era valorizada a ausculta cardíaca, medidas antropométricas (peso e altura) e da pressão arterial (PA). Concomitante ao exame médico, o responsável pelo aluno preenchia um questionário cuja meta era a obtenção de informações sobre moléstias cardiovasculares, doenças hipertensivas e ou morte súbita em familiares.

Quanto aos parâmetros laboratoriais, foram considerados, como dentro dos limites normais para o sexo e idade, segundo o Comitê de Aterosclerose e Hipertensão na Infância do Conselho de Doença Cardiovascular do Jovem e Comitê de Nutrição da Associação Americana de Cardiologia ${ }^{8}$, os valores: colesterol total: até $180 \mathrm{mg} \%$; triglicerídios: até 140mg\%; HDL-colesterol: $40 \mathrm{mg} \%$ ou acima; LDL-colesterol: até $130 \mathrm{mg} \%$.

Quanto à conceituação de pressão arterial sistólica (PAS) e diastólica (PAD) foram seguidos os critérios do Report of the Second Task Force on Blood Pressure Control in Children ${ }^{9}$ : normal - PAS e ou PAD entre o percentil 90 e 95 ; HA - percentil 95 ou acima.

Considerou-se a presença de obesidade quando o índice de massa corporal estava acima do percentil 95 para a idade e sexo ${ }^{10}$.

Para a análise estatística, os dados foram tabelados em protocolo específico para análise individualizada e realizadas medidas de tendência central e utilizados recursos da estatística inferencial, através do teste do qui-quadrado para dados qualitativos. Para o cálculo do $\mathrm{n}$ foi feita estimativa por proporção populacional. Foi estipulado o nível de significância de $5 \%(0,05)$ como alfa crítico e valores de $\mathrm{p}$ $<0,05$ foram referidos quando identificados.

\section{Resultados}

A idade média foi de 10 anos e 5 meses \pm 2 anos e 8 meses. Eram do sexo feminino $796(53,03 \%)$ alunos e do masculino 705 (46,97\%). A cor branca 1497 (99,74\%) predominou nitidamente. $\mathrm{O}$ peso médio foi de $38,62 \% \pm 13,22 \mathrm{~kg}$ e a altura média de $142,5 \pm 15,06 \mathrm{~cm}$.

A renda familiar mostrou que 305 (37,29\%) famílias dos colégios da rede pública ganhavam menos de três salários mínimose 38 (4,65\%) percebiam acima de 16 salários mínimos; por outro lado, em relação aos colégios particulares esta relação praticamente se inverteu, isto é, foram encontradas 40 $(5,86 \%)$ famílias que ganhavam menos de três salários mínimose $260(38,07 \%$ ) com 16 ou mais salários mínimos.

Os níveis séricos do colesterol mostraram uma ampli-

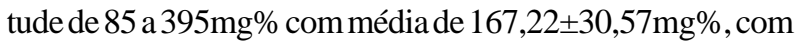
intervalo de confiança de 165,7 a $168,77 \mathrm{mg} \%$. A mediana foi de $165 \mathrm{mg} \%$ e a moda de $145 \mathrm{mg} \%$.

Foram detectadas $420(27,98 \%)$ crianças com colesterolemia $>180 \mathrm{mg} \%$, sendo que $189(12,6 \%$ ) possuíam o colesterol $>200 \mathrm{mg} \%, 72(4,8 \%)$ apresentavam a colesterolemia $>220 \mathrm{mg} \%$ e $24(1,59 \%)>240 \mathrm{mg} \%$ (fig. 1 ).

A prevalência de colesterol $>180 \mathrm{mg} \%$ em colégios particulares foi de $227(15,12 \%)$ crianças e de $193(12,86 \%)$ nos colégios públicos $\left(\mathrm{x}^{2}=17,17 \mathrm{p}<0,001\right)$. 
APAS teve como média $101,94 \pm 14,63 \mathrm{mmHg}$, com intervalo de confiança de 101,20 a 102,68mmHg. A média da PAD foi de $56,22 \pm 14,07 \mathrm{mmHg}$, com intervalo de confiança de 55,86 a 56,58mmHg. Foram encontradas 75 (5\%) crianças comPAS acima do percentil 95 e, 48 (3,20\%) com PAD acima do percentil 95 .

Em relação ao peso, $111(7,40 \%)$ crianças apresentaram-no elevado, não havendo diferença estatística entre os colégios. Vinte e sete $(1,8 \%)$ crianças apresentaram HA sistólica e hipercolesterolemia, sem diferença estatística entre os colégios. Crianças com HA diastólica e hipercolesterolemia foram em número de 23 (1,53\%), também sem diferença estatística.

As crianças que apresentaram obesidade não mostraram uma maior prevalência de hipercolesterolemia. O teste de correlação de Pearson não mostrou correlação entre as variáveis peso e colesterolemia $(r=0,09)$.

A história familiar positiva (morte súbita, dislipidemias e ou doença cardíaca isquêmica) foi encontrada em 161 $(38,33 \%)$ crianças que apresentaram hipercolesterolemia concomitante. Entretanto, 259 (61,67\%) crianças com hipercolesterolemia não tinham história familiar positiva.

A trigliceridemia teve como média $89,45 \pm 36,82 \mathrm{mg} \%$, com intervalo de confiança de 87,59 a $91,31 \mathrm{mg} \%$. A hipertrigliceridemia foi encontrada em 136 (9,06\%) alunos. Não houve diferença estatística entre os diversos colégios. A hipertrigliceridemia associada a hipercolesterolemia foi observada em 70 escolares. A probabilidade de escolares com hipertrigliceridemia estar também com hipercolesterolemia mostrou significância estatística $(\mathrm{p}<0,001)$.

A lipoproteína de baixa densidade (LDL-colesterol) teve a média de $97,27 \% \pm 23,33 \mathrm{mg} \%$ com intervalo de confiança de 95,89 a 98,65\%. Cento e cinqüenta e cinco (10,33\%) alunos apresentaram oLDL-colesterol elevado (>130mg\%), correspondendo a $36,90 \%$ da amostra de 420 crianças que apresentaram hipercolesterolemia simultaneamente. O teste de correlação de Pearson entre o LDL-colesterol e a hipercolesterolemia mostrou a existência de uma associação muito forte $(r=0,93)$. $O$ coeficiente de determinação $\left(r^{2}\right)$ foi de 0,87 .

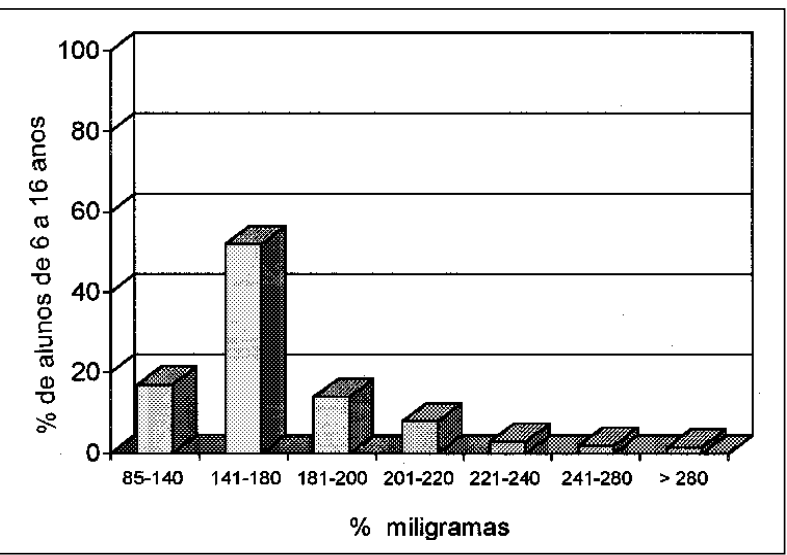

Fig. 1 - Níveis de colesterol sérico dos escolares de Bento Gonçalves.

\begin{tabular}{|c|c|c|}
\hline \multicolumn{3}{|c|}{ Tabela I - Associação de fatores de risco na amostra } \\
\hline Fatores de risco associados & $\mathrm{n}$ & $\%$ \\
\hline \multicolumn{3}{|l|}{ Hipercolesterolemia } \\
\hline \multicolumn{3}{|l|}{ HDL-colesterol baixo } \\
\hline LDL-colesterol elevado & 20 & 1,33 \\
\hline \multicolumn{3}{|l|}{ Hipercolesterolemia } \\
\hline Hipertensão diastólica & 19 & 1,27 \\
\hline \multicolumn{3}{|l|}{ Hipercolesterolemia } \\
\hline $\begin{array}{l}\text { Hipertensão arterial } \\
\text { sistólica e diastólica }\end{array}$ & 7 & 0,47 \\
\hline \multicolumn{3}{|l|}{ Hipercolesterolemia } \\
\hline \multicolumn{3}{|l|}{ Ambas as pressões elevadas } \\
\hline LDL-colesterol elevado & 2 & 0,13 \\
\hline \multicolumn{3}{|l|}{ Hipercolesterolemia } \\
\hline \multicolumn{3}{|l|}{ HDL-colesterol baixo } \\
\hline \multicolumn{3}{|l|}{ LDL-colesterol elevado } \\
\hline Hipertrigliceridemia & 10 & 0,67 \\
\hline \multicolumn{3}{|l|}{ Hipertensão diastólica } \\
\hline $\begin{array}{l}\text { (*) Vê-se que } 1,93 \% \text { possuíar } \\
\text { sentavam } 4 \text { fatores de riscos }\end{array}$ & ric & $0,67 \%$ apre- \\
\hline
\end{tabular}

A lipoproteína de alta densidade (HDL-colesterol) teve a média de $52,07 \pm 10,49 \mathrm{mg} \%$ com intervalo de confiança de 51,54 a 52,60mg\%. Cento e quarenta e seis $(9,73 \mathrm{mg} \%)$ crianças estavam com HDL-colesterol <40mg\%. Destas, 31 $(2,07 \%)$ mostraram-se com hipercolesterolemia associada. Portando, $115(7,66 \%)$ escolares tinham HDL-colesterol baixo e colesterolemia normal.

Na amostra, 1,93\% dos estudantes apresentaram três fatores de risco para aterosclerose associados e $0,67 \%$ mostraram-se com quatro fatores de risco em concomitância (tab. I).

\section{Discussão}

Em Bento Gonçalves há 11.793 estudantes nas escolas de $1^{\circ}$ grau. Deste total, 1501 alunos participaram do estudo, sendo que 683 pertenciam a colégios particulares e 818 a colégios da rede pública. A participação de cada aluno ocorreu de acordo com a autorização dos responsáveis, o que nos levou a crer que só participariam da pesquisa aqueles que tivessem história familiar positiva para distúrbios cardíacos, o que não foi constatado, já que somente 38,33\% das famílias tinham ou sabiam de sua história familiar. Outrossim, os 1501 alunos correspondiam a 12,73\% dos estudantes da cidade. O tamanho mínimo da amostra calculado para que houvesse significância estatística foi de 478 pessoas. Portanto, esta amostragem corresponde a cerca de três vezes o valor do n calculado $(a=5,0 \%)$.

A morbimortalidade por doença cardiovascular constitui um dos grandes desafios da medicina contemporânea. Apesar da enorme aquisição de conhecimentos na área da cardiologia, ainda é a doença cardiovascular a maior causa de óbito dos países desenvolvidos ${ }^{11,12}$. No momento os três fatores de risco mais importantes para o desenvolvimento da aterosclerose são, pela ordem, o colesterol, o hábito de 
fumar e a HA. A redução da hipercolesterolemia reduz em $30 \%$ a ocorrência de aterosclerose coronária, enquanto que a suspensão do hábito de fumar e a normalização da PA a reduzem em $24 \%$ e $8,9 \%$, respectivamente ${ }^{13}$.

Os fatores de risco para a aterosclerose, avaliados no presente estudo, foram o colesterol total e frações, os triglicerídeos, a HA, a obesidade, a história familiar e o nível socioeconômico. O hábito de fumar, um dos mais importantes fatores de risco aterosclerótico, não foi relacionado, por terem todos os participantes negado tal conduta.

Em relação ao sexo, não houve diferença na participação de meninos e meninas. Denota-se neste estudo a grande prevalência de estudantes brancos $(99,74 \%)$. Dennison e $\mathrm{col}^{23}$ mostraram que há diferença entre os grupos raciais. Os negros, de modo geral, têm colesterol mais elevado, às custas do HDL-colesterol.

A renda familiar entre os colégios públicos e particulares, utilizada neste trabalho como indicador do nível socioeconômico, mostrou diferença importante. A renda familiar mostrou-se elevada em relação ao resto do país, mesmo em educandários públicos, e talvez, por isso, estudos feitos em outros locais (cidades e regiões) possam mostrar uma prevalência de fatores de risco diferente da encontrada neste estudo.

Na presente pesquisa, o fator socioeconômico elevado mostrou-se relevante em relação ao colesterol e LDLcolesterol, não acontecendo o mesmo quanto à obesidade, PA, HDL-colesterol e triglicerídeos.

Observou-se que a freqüência de obesidade foi relativamente baixa $(6,33 \%)$, tanto no subgrupo de renda mais alta (colégios particulares, 3,0\%) como no de renda mais baixa (colégios públicos, 3,33\%). É importante o controle de peso durante a infância, haja vista que a obesidade adquirida neste período da vida tende a persistir na vida adulta ${ }^{14}$. A obesidade não é considerada um fator de risco direto para a aterosclerose coronária mas, em geral, vem acompanhada de HA e diabetes mellitus ${ }^{15}$. O obeso teria maior incidência de angina e insuficiência cardíaca congestiva. Assim, através do controle de peso, pode-se potencialmente diminuir a prevalência de, no mínimo, dois fatores de risco notórios, a saber: o diabetes mellitus e a HA.

Um fato importante, encontrado na pesquisa, foi a constatação de que os fatores de risco para a aterosclerose e posterior DAC, realmente estão presentes desde a infância. O estudo de Framingham indicou uma contínua elevação de risco para DAC à medida que o colesterol ultrapassa o nível de $180 \mathrm{mg} \%{ }^{16}$. Em países em que a incidência de coronariopatia é muito baixa, a concentração média do colesterol em adultos está entre 140 e $160 \mathrm{mg} \%{ }^{17}$. Talvez esses níveis sejam os ideais para a prevenção de uma posterior coronariopatia na vida adulta. No Japão, por exemplo, a incidência de coronariopatia é de 1/8 em relação aos Estados Unidos, em homens adultos de 55 a 60 anos, ressaltando-se que o colesterol sérico nos americanos com aquelas idades, varia de 240 a $260 \mathrm{mg} \%$, enquanto que no Japão, nas mesmas idades, o nível situa-se entre 140 e $160 \mathrm{mg} \%{ }^{18}$.

O estudo de Bogalusa ${ }^{19}$ revelou que a ateromatose da íntima da aorta foi demonstrada mesmo com níveis de 140 a $170 \mathrm{mg} \%$. A meta de epidemiologistas dos Estados Unidos é de que as crianças norte-americanas atinjam uma média de colesterol plasmático de $150 \mathrm{mg} \%$. Em outro importante estudo, o de Muscatine ${ }^{20}, 24 \%$ das crianças possuíam o colesterol $>200 \mathrm{mg} \%, 9 \% \geq 220 \mathrm{mg} \%, 3 \% \geq 240 \mathrm{mg} \%$ e, $1 \%$ $\geq 260 \mathrm{mg} \%$. Em nosso estudo, percebe-se que 420 crianças possuíam colesterol total $>180 \mathrm{mg} \%$, correspondendo a $27,98 \%$ dos estudantes examinados (cerca de um em cada quatro escolares ).

Sabe-se que quanto maior o valor da colesterolemia maior é o risco de coronariopatia precoce. Não se pode descartar a possibilidade de que as crianças de nosso estudo, com colesterol $>180 \mathrm{mg} \%$, possam ser consideradas eventuais candidatas a uma futura coronariopatia. Somente estudos longitudinais poderão indicar a relativa permanência do colesterol nas mesmas concentrações. Mesmo que apenas uma parcela venha a permanecer nos níveis de risco, isto por si só já se constituiria em um número significativo de crianças com um fator preditivo para a aterosclerose identificado precocemente na vida.

Atitudes terapêuticas neste subgrupo específico, visando a diminuição da colesterolemia, certamente deverão se constituir em ações preventivas a serem avaliadas no futuro.

A HA, importante fator de risco coronário, é superada somente pela hipercolesterolemia. A HA sistólica está mais relacionada ao acidente vascular cerebral ${ }^{21}$ do que à coronariopatia: inversamente ocorre em relação a HA diastólica. Segundo Castelli, tanto a PAS como a PAD podem ser consideradas como fatores de risco coronário ${ }^{22}$.

Na literatura, a prevalência de HA varia de 1,4 a $11 \%$ 23,24. No presente trabalho, a HA diastólica foi observada em 48 crianças $(3,20 \%)$. Não houve significância estatística entre os colégios quanto a PA.

Estudos ecocardiográficos nas crianças de Bogalusa ${ }^{19}$ e de Pennock e col ${ }^{25}$ mostraram um aumento contínuo da espessura da parede ventricular posterior esquerda em paralelo aos níveis crescentes da pressão sangüínea. Existe uma correlação significativa entre a medida da PA inicial e as medidas subseqüentes, em crianças acompanhadas durante vários anos ${ }^{26}$.

As crianças que são classificadas em um canal, como por exemplo, o percentil 95 , tendem a permanecer no mesmo com o decorrer do tempo. Exemplificando, em uma criança de 14 anos de idade e no percentil 95, uma PA de 128/ $78 \mathrm{mmHg}$ poderá ser equivalente a $180 / 110 \mathrm{mmHg}$ na idade adulta ${ }^{27}$. No adulto, a HA está relacionada com a mortalidade e a morbidade por doença cardíaca coronária ${ }^{28}$.

A história familiar foi positiva em 161 (38,33\%) escolares com hipercolesterolemia, o que significa que aproximadamente dois terços das crianças com hipercolesterolemia não apresentavam história familiar positiva para distúrbios cardíacos, hipertensão, morte súbita ou dislipidemias. Quando a história familiar é positiva, há grande possibilidade do colesterol estar $>180 \mathrm{mg}^{29}$.

Dennison e col $^{30}$ encontraram somente $40 \%$ das crianças brancas e $21 \%$ das negras que apresentavam hiperco- 
lesterolemia com história familiar de doença cardiovascular. Estes achados indicam que mesmo sem história familiar de doenças cardiovasculares, o fator de risco pode estar presente e, por isso, é recomendável pesquisá-lo.

A hipertrigliceridemia é relacionada positivamente com o risco de DAC, mas quando o HDL-colesterolé elevado, este risco é neutralizado ${ }^{31}$. Os triglicerídeos, no presente trabalho, não mostraram sofrer influência em relação ao nível socioeconômico.

O LDL-colesterol correlaciona-se com o colesterol total ( $r=0,84$ para o sexo masculino e $r=0,88$ para o feminino ), sendo um poderoso preditor de pessoas de risco, em qualquer idade ${ }^{32}$. Em nosso estudo, não foi encontrada nenhuma criança com LDL-colesterol $>130 \mathrm{mg} \%$ e que, ao mesmo tempo, apresentasse a colesterolemia normal.

OHDL-colesterol carreia 1/4 do colesterol sérico. Tem sido demonstrado que quanto maior o nível dessa lipoproteína, menor é o risco de aterosclerose e suas conseqüências ${ }^{33}$. O HDL-colesterol, no nosso estudo, mostrou-se $<40 \mathrm{mg} \%$ em concomitância com o colesterol $>180 \mathrm{mg} \%$ em $31(2,07 \%)$ crianças. Entretanto, encontrou-se o HDLcolesterol em níveis baixos com o colesterol total normal em $115(7,66 \%)$ crianças. É relevante comentar que a dosagem pura e simples do colesterol total para efeito de rastreamento de fator de risco coronário, não descobriria o HDL-colesterol em níveis baixos, que por si só é um fator de risco ${ }^{34}$.

A aterosclerose e a conseqüente $\mathrm{DAC}$, em particular, parece ter uma origem multifatorial. As variáveis de risco vão se somando, aumentando a probabilidade de coronariopatia. Foram encontrados 1,93\% dos escolares com três fatores de risco associados e $0,67 \%$ com quatro fatores de risco.

A pesquisa e a análise dos fatores de risco em cada região e até mesmo em cada cidade, deveria registrar a prevalência dos fatores de risco para a aterosclerose na infância. Isso representa um passo importante para a identificação de uma população de risco, tornando-se necessário o seu seguimento e possíveis medidas intervencionistas ${ }^{35}$. Existem trabalhos ${ }^{36}$ que comprovaram a possibilidade de estabilização e mesmo de regressão das lesões ateroscleróticas já existentes.

Quanto maior a prevalência de fatores de risco, maioréa incidência de um estado mórbido ${ }^{37}$. A identificação de crian- ças com fatores de risco, em adição aos benefícios que elas passam a usufruir, pode também permitir a descoberta de outros membros da família portadores de fatores de risco, até então ignorados, devido à comprovada agregação familiar ${ }^{38}$.

Atualmente são feitos testes de triagem para grande número de doenças, visando a descoberta precoce e o maior êxito terapêutico. Assim, a fenilcetonúria ocorre em 1/ 14.000 neonatos e o hipotireoidismo em torno de 1/4.000. Como, então, não rastrear precocemente os riscos potenciais para as doenças cardiovasculares, que são a maior causa de morbidade e mortalidade deste século?

O estudo de crianças assintomáticas forma uma ótima base para a cardiologia preventiva e, indubitavelmente, terá um grande impacto no decréscimo da doença cardiovascular no futuro. O pediatra deve ser um dos profissionais de saúde responsável pela investigação dos fatores de risco para a aterosclerose na infância.

Em conclusão, este trabalho demonstra que: 1) os fatores de risco para a aterosclerose e DAC são prevalentes na infância; 2) dentre os fatores de risco, o de maior prevalência é a hipercolesterolemia isolada; 3) a probabilidade de encontrar mais de um fator de risco é maior do que um fator de risco isoladamente; 4) a colesterolemia total dentro da normalidade não exclui alteração nas lipoproteínas; 5) na amostra estudada, é possível afirmar que o nível socioeconômico influiu significantemente na prevalência de hipercolesterolemia e de aumento de LDL-colesterol; o mesmo não acontecendo em relação aos triglicerídeos, HDL- colesterol, PA, obesidade e história familiar; 6) embora a presença de uma história familiar positiva de morte súbita, dislipidemiae doença isquêmica tenha mostrado associação significante com hipercolesterolemia, sua ausência não elimina a possibilidade da existência de fatores de risco para a aterosclerose na infância; 7) a probabilidade de escolares de Bento Gonçalves apresentarem hipercolesterolemia é de um para quatro; 8) há necessidade de serem desenvolvidos outros estudos populacionais dos fatores de risco para a aterosclerose na idade pediátrica, visando sua detecção precoce, em função de possíveis diferenças regionais; 9) o $1^{\circ}$ sinal de doença cardíaca aterosclerótica no adulto pode ser a morte súbita. Considerando que os fatores de risco que a precede têm início durante a infância, a sua prevenção passa a ser uma atribuição do pediatra.

\section{Referências}

1. Mc Intosh HD - Risk factors for cardiovascular disease and death: a clinical perspective. J Am Coll Cardiol 1989; 14: 24-30.

2. Puska P- Possibilities of a preventive approach to coronary heart disease starting in childhood. Acta Paediat Scan 1986; 318(suppl): 229-33 .

3. Cresanta JL, Burke GL, Downey AM, Freedman DS, Berenson GS - Prevenção da aterosclerose na infância. Clínicas Pediátricas da América do Norte 1986; 4 : 879-903.

4. Zeek P - Juvenile arteriosclerosis. Arch Pathol 1930; 10: 417-46.

5. Holman RL, McGill Jr HC, Strong JP, Geer JC- The natural history of atherosclerosis. Am J Path 1958; 34: 209-35.
6. Angelini A, Thiene G, Frescura C, Beroldi G-Coronary arterial wall and atherosclerosis in youth (1-20 years): a histologic study in a Northern Italy population. Int J Cardiol 1990; 28: 361-70.

7. Secretaria da Educação e Cultura de Bento Goncalves, oficio AT/INF/584-90 de 18/10/1990.

8. Cresanta JL, Srinivasan SR, Webber LS, Berenson G-Serum lipid and lipoprotein cholesterol grids for cardiovascular risk screening of children. Am J Dis Child 1984; 138: 379-87.

9. Horan MJ - Report of second task force on blood pressure control in children. Pediatrics 1987; $7:$ 1-25. 
10. Weidman W, Kwiterovich Jr P, Arky R et al - Diagnosis and treatment of primary hyperlipidemia in childhood. Circulation 1986; 74: 1181A-8A.

11. Bush TL, Riedel D - Screening for total cholesterol - Do the National Cholesterol Education Program's Recomendations Detected Individuals at High Risk of Coronary Heart Disease? Circulation 1991; 83: 1287-93.

12. Laurenti R - Aterosclerose: importância, aspectos epidemiológicos. Estudo central do problema no Brasil e em São Paulo. Cardiologia 1987; 1: 162-9.

13. Sytkowski PA, Kannel WB, D'Agostino RB - Changes in risk factors and the decline in mortality from cardiovascular disease. The Framingham Heart Study. N Engl J Med 1990; 322: 1635-41

14. Kannel WB, Mcgee D, Gordon T - A general cardiovascular risk profile: the Framinghan Study. Am J Cardiol 1976; 38: 46-51.

15. Okumiya N, Tanaka K, Ueda K, Omae T - Coronary atherosclerosis and antecedents risk factors:pathologic and epidemiologic study in Hisayama. Jpn Am J Cardiol 1985; 56: 62-6.

16. Castelli WP - Epidemiology of coronary heart disease: The Framinghan Study Am J Med 1984: 4-12.

17. Tanaka K, Masuda J, Imamura Tetal - A nation-wide study of atherosclerosis in infants, children and young adults in Japan. Atherosclerosis 1988; 72: 143-56.

18. Robertson TL, Kato H, RKhoads GG, Kagan A, Marmot M - Epidemiologic Studies of coronary heart disease and stroke in Japanese men living in Japan, Hawaii and California. Am J Cardiol 1977; 39: 239-43

19. Srinivasan SR - Racial (black-white) differences in serum lipoprotein(a) - distribution and its relation to parental myocardial infarction in children - Bogalusa Heart Study. Circulation 1991; 84: 160-7.

20. Lauer R, Connor WE, Leaverton PE, Reiter MA,Clarke WR-Coronary heart disease risk factors in school children: The Muscatine Study. J Pediatr 1975; 86: 697-706.

21. Burke GL, Voors AW, Shear CL et al-Blood pressure pediatrics, Bogalusa Heart Study. Pediatric 1987; (suppl): 784-8

22. Voors A., Webber L, Berenson G - Epidemiologia da hipertensão essencial no jovem. Implicações para a prática clínica. Clínicas Pediátricas da América do Norte 1978: 15-27.

23. Moss AJ - Métodos indiretos de medida da pressão sangüínea. Clínicas Pediátricas da América do Norte 1978: 3-14.

24. Zinner SH, Rosner B, Oh W, Kass EH-Significance of blood pressure in infancyfamilial aggregation and predictive effect on latter blood pressure. Hypertension $1985 ; 7: 411-6$
25. Pennock LW, Fixler DE - Left ventricular hipertrophy in adolescentes with elevated blood pressure: assesment bky chest roentgenography, electrocardiography, echocardiography. Pediatrics 1981; 67: 255-9.

26. Heyden S, Bartel AG, Hames CG, McDonovah JR - Elevated blood pressure levels in adolescents. Evans Couty Georgia. JAMA 1969; 209: 163-8.

27. Clarke WR, Schrott HG, Leaverton PE, Connor WE, Lauer RM - Tracking of blood lipids and blood pressures in school age children: The Muscatine Study. Circulation 1978; 58: 626-33.

28. Sorlie PD, Garcia-Palmieri MR, Castillo-Staab I, Costas Jr R, Oalmann M, Havlik R - The relation of antemortem factors to atherosclerosis at autopsy. Am J Pathol 1981; 103: 345-52.

29. Hennekens CH, Jesse MJ, Klein BE, Gourley JE, Blumenthal S - Cholesterol among children of men with myocardial infarction. Pediatrics 1976; 58: 211-7.

30. Dennison BA, Kikuchi DA, Srinivasan SR, Webber LS, Berenson GS - Parental history of cardiovascular disease as indication for screening for lipoprotein abnormalities in children. J Pediatr 1989; 115: 186-94.

31. Blumenthal S, Jesse MJ, Hennekens CH, Klein BAE, Ferrer PL, Gourley JERisk factors for coronary artery disease in children of affected families. J Pediatr $1975 ; 1187-92$.

32. Lee J, Lawer RM - Aspectos pediátricos da aterosclerose e da hipertensão. Clínicas Pediátricas da América do Norte. Cardiol Pediátrica 1978; 913-33.

33. Salomen J - HDL, HDL2 and HDL3, subfractions and the risk of acute miocardial infarction. Circulation 1991; 84: 129-39.

34. Gordon DJ, Knoko J, Probstfield JL, Superko R, Tyroler HA - For the Lipids Research Clinics Program - High-Density Lipoprotein Cholesterol and Coronary Heart Disease in Hipercholesterolemic Men: The Lipid Research Clinics Coronary Primary Prevention Trial. Circulation 1986; 74: 1217-25.

35. Oganov RG, Tubol IB, Zhukoskii GS et al - Epidemilogical characteristics of dislipoproteinemia and certain other risk factors of atherosclerosis and ischemia heart disease in 11 - and - 14 year children in different climatogeografic zones Results of a Cooperative Study. Cor Vasa 1988; 30: 248-56.

36. Armstrong ML, WarnerEO, Connor WE-Regression of coronary atheromatosis in Rhesus Monkeys Circ Res 1970; 27: 59-67.

37. Fletcher RH, Fletcher SW, Wagner EH -Epidemiologia Clínica 1989; 126-44.

38. Bucher KD, Schrott HG, Clarke WR, Lauer RM - The Muscatine Cholesterol Family Study: Familial aggregation of blood lipids and relationship of lipid levels to age sex and hormone use. J Chronic Dis 1982; 35:375-84 